\title{
'Double - Arch' / Cumbo's Sign - Pathognomonic for Ruptured Hydatid Cyst in Lung
}

\author{
ANIRBAN DAS, ${ }^{1}$ ANIRBAN SARKAR, ${ }^{2}$ SIBES KUMAR DAS ${ }^{3}$
}

\section{Pictorial CME:}

A 35 - years old non - smoker, male farmer presented with right sided, intermittent, dull aching chest pain with occasional dry cough. History of exposure to pet cats was present. Chest radiograph - posteroanterior view showed a cystic lesion containing air and fluid, which was capped by a crescent of air in right lower zone - the 'double arch' or Cumbo's sign, pathognomonic for ruptured hydatid cyst in lung. Peripheral blood eosinophil count was $1,800 / \mathrm{cmm}$. Anti - echinococcal antibody was strongly positive. The patient was treated by surgical resection of ruptured cyst without any recurrence during next six months follow up.

Hydatid cyst is caused by the parasite Echinococcus granulosus. Liver is the most common site of involvement, followed by lung. Isolated pulmonary involvement is rare. Intact cyst in lung is diagnosed easily by radiology and serology, but rupture and subsequent infection change the scenario, and often misdiagnosed as mass lesion. In such cases, different radiological signs provide the clue, which is subsequently confirmed by serology. Among them, Double - arch sign or Cumbo's sign is pathognomonic for the diagnosis of ruptured hydatid cyst in lung. Air entry between pericyst and endocyst due to rupture of cyst wall, produces the 'meniscus' or 'crescent' sign. ${ }^{1}$ With further entry of air in between two, shrinking cyst ruptures, and fluid is coming out from endocyst with entry of some air into endocyst. So that endocyst containg air - fluid level, capped by a crescent

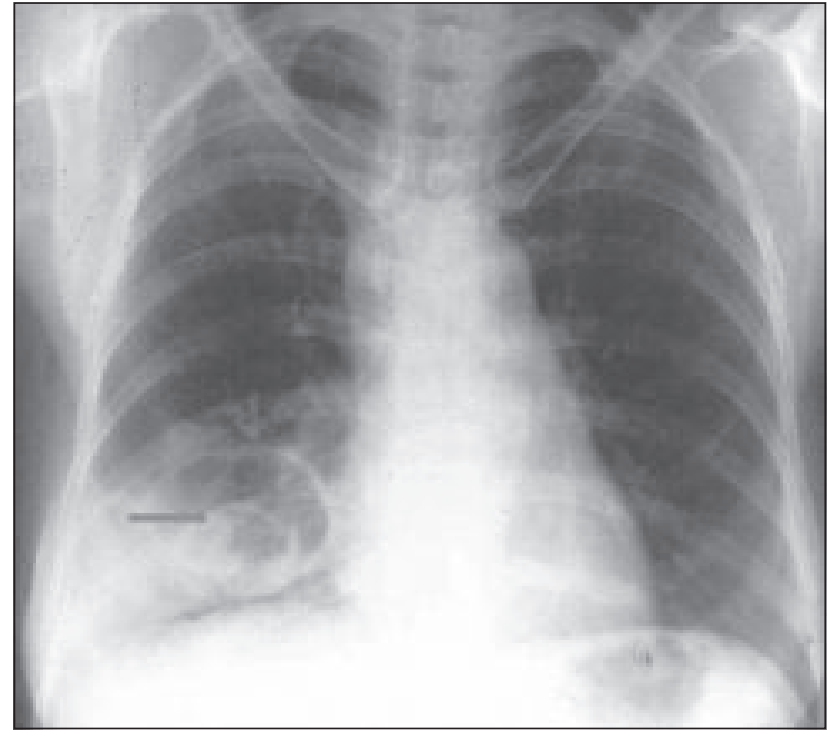

Fig.-1: CXR - PA view showing 'Double-arch'sign (violet \& pink arrow).

of air within pericyst and endocyst produces 'double arch' sign or Cumbo's sign. ${ }^{2}$

\section{References:}

1. Beggs I. The radiology of hydatid disease. Am J Roentgenol 1985; 145: $639-648$.

2. Balikian JP, Mudarris FF. Hydatid disease of the lungs: a roentgenologic study of 50 cases. Am J Roentgenol 1974;122:692-707.

1. Assistant Professor, Department of Pulmonary Medicine, Medical College, Kolkata, West Bengal, India, E-mail: dranirbandas_chest@rediffmail.com

2. Assistant Professor, Department of Pulmonary Medicine, Medical College, Kolkata, West Bengal, India, E-mail: anirbansrkr10@gmail.com

3. Professor, Department of Pulmonary Medicine, Medical College, Kolkata, West Bengal, India, E - mail: sibesdas67 @gmail.com

Correspondence : Dr. Anirban Das, Assistant Professor, Department of Pulmonary Medicine, Medical College, Kolkata, West Bengal, India, E-mail: dranirbandas_chest@rediffmail.com 